\title{
Relato de experiência da atuação da terapia ocupacional em grupo de indivíduos reumáticos
}

\author{
A report of an experience of occupational therapy \\ in a group with individuals with \\ rheumatic diseases \\ Johanna Noordhoek ${ }^{1}$, Maria Cristina de Oliveira Silva ${ }^{2}$, \\ Aline Torquetti ${ }^{3}$, Ligia de Loiola Cisneros ${ }^{4}$
}

\begin{abstract}
NOORDHOEK, J.; SILVA, M. C. de O.; TORQUETTI, A.; CISNEROS, L. de L. Relato de experiência da atuação da terapia ocupacional em grupo de indivíduos reumáticos. Rev. Ter. Ocup. Univ. São Paulo, v. 20, n. 1, p. 13-19, jan./abr. 2009.

RESUMO: O indivíduo com doença reumática apresenta alterações fisiológicas e anatômicas que interferem na performance durante as tarefas cotidianas. A terapia ocupacional, atuando na promoção da saúde dessa população, visa propiciar melhoras funcionais, sociais e emocionais, favorecendo o desempenho de papéis e o aumento da qualidade de vida. O objetivo deste trabalho é descrever a experiência da atuação da terapia ocupacional em um grupo de orientação a indivíduos com doenças reumáticas. As proposições expostas baseiam-se na experiência empírica do Grupo de Orientação aos Indivíduos Acometidos por Doenças Reumáticas, projeto de extensão da Universidade Federal de Minas Gerais. O processo educativo é ofertado através de oficinas terapêuticas, tendo por base o uso de atividades lúdico-recreativas. Essa estratégia possibilita a educação, socialização e o desenvolvimento de habilidades para o enfrentamento da doença e favorece o desempenho de papéis ocupacionais.
\end{abstract}

DESCRITORES: Promoção da saúde. Terapia ocupacional. Doenças reumáticas.

\footnotetext{
1. Professora Assistente do Departamento de Terapia Ocupacional da Escola de Educação Física, Fisioterapia e Terapia Ocupacional da Universidade Federal de Minas Gerais (UFMG).

2. Professora Assistente do Departamento de Terapia Ocupacional da Escola de Educação Física, Fisioterapia e Terapia Ocupacional da Universidade Federal de Minas Gerais (UFMG).

3. Terapeuta Ocupacional.

4. Professora Adjunta do Departamento de Fisioterapia da Escola de Educação Física, Fisioterapia e Terapia Ocupacional da Universidade Federal de Minas Gerais (UFMG).

Endereço para correspondência: Professora Johanna Noordhoek. Rua Mármore, $\mathrm{n}^{\circ}$ 362/404, Bairro Santa Teresa, CEP: 31.010-220. Belo Horizonte, Minas Gerais. E-mail: johannanoord@yahoo.com.br
} 
NOORDHOEK, J. et al. Relato de experiência da atuação. Rev. Ter. Ocup. Univ. São Paulo, v. 20, n. 1, p. 13-19, jan./abr. 2009.

\section{INTRODUÇÃO}

s doenças reumáticas compreendem um gru-
po de doenças crônicas onde há o predomí-
nio da dor e da rigidez no aparelho locomotor (MOREIRA; CARVALHO, 1996). Os indivíduos acometidos apresentam como queixa principal a dor, sendo essa de curso crônico, podendo alterar o sono, a concentração, levar a uma diminuição da mobilidade e à incapacidade para o trabalho, motivação para a recreação, além de afetar o estado emocional do indivíduo (TROMBLY, 2005).

Diante do caráter crônico das doenças reumáticas e das limitações impostas no cotidiano dos indivíduos, torna-se necessário criar estratégias que minimizem os impactos sobre a qualidade de vida dos envolvidos. Nesse sentido, a Terapia Ocupacional objetiva a promoção e/ ou restabelecimento do desempenho funcional nas ocupações que compõe o cotidiano dos indivíduos, trabalhando em todos os níveis de atenção à saúde. Para Hahn (1995), quando o foco de atuação se volta para a promoção da saúde, a Terapia Ocupacional dirige-se a gerar condições e modo de vida que sejam sadios, estimuladores, prazerosos e agradáveis.

A promoção de saúde, segundo Souza e Grundy (2004), é um processo abrangente e contínuo que envolve prevenção, educação e participação de diferentes setores da sociedade na elaboração de estratégias que permitam a efetividade da educação para a saúde. De acordo com Czeresnia (1999), as ações de promoção de saúde são caracterizadas por visar o aumento da saúde e do bem estar dos indivíduos.

Acredita-se que um padrão natural e equilibrado de ocupações promove saúde e qualidade de vida e que a educação para o cidadão com doenças reumáticas é uma estratégia eficaz para a promoção da saúde (CARDOSO, 2005).

O indivíduo acometido por doenças reumáticas geralmente não avalia como seu próprio comportamento pode modificar a sua condição de saúde, ou como o comportamento pode ser modificado para auxiliar sua recuperação (POLLEY; HUNDER, 1980). De acordo com Law (1998, 2002), a participação efetiva e autônoma na vida é uma influência positiva na saúde e bem estar do indivíduo. $\mathrm{O}$ engajamento de adultos e idosos, em atividades de lazer e recreação, auxilia no desenvolvimento de uma rede social de suporte, além de promover saúde e bem estar.

Os programas de educação para reumáticos objetivam alcançar e manter uma mudança comportamental através de metodologias voltadas à conquista, pelos indivíduos, de hábitos e métodos próprios de gerenciamento dos seus problemas. Em geral, esses programas promovem o fornecimento de informações básicas sobre a doença, apontando possibilidades de gerenciar a dor, a instabilidade articular, a fraqueza muscular e a fadiga. Fornecem, ainda, orientações quanto à execução de atividades de vida diárias, de modo a evitar a dor e reduzir o estresse nas articulações acometidas (TAAL et al., 1997) Barry et al. (1994) e Barlow et al. (1998); Hammond et al. (1994), Lindroth et al. (1997) e Helliwell et al. (1999).

Vários estudos, de natureza quantitativa, atestam a eficácia dos programas educacionais para essa população, em termos de benefícios físicos e psíquicos, alcançados pelos pacientes (SHOLTEN et al., 1999; BARLOW et al., 2000; HAMMOND; FREEMAN, 2001). Esses programas são capazes de melhorar a habilidade funcional pela estimulação do aumento da percepção de auto-eficácia dos indivíduos (TAAL et al., 1997).

O Grupo de Orientação aos Indivíduos Acometidos por Doenças Reumáticas (GOIADR), foi criado com os objetivos de educar, socializar, melhorar o desempenho de papéis ocupacionais e desenvolver habilidades pessoais e novas possibilidades de enfrentamento da doença (NOORDHOEK et al., 2005).

O objetivo deste trabalho é descrever a experiência da atuação da terapia ocupacional neste grupo de orientação a indivíduos com doenças reumáticas.

\section{Metodologia da intervenção grupal}

O GOIADR foi montado a partir da iniciativa de uma docente do Departamento de Terapia Ocupacional, numa parceria com o Departamento do Aparelho Locomotor e com o Serviço de Reumatologia da Faculdade de Medicina da Universidade Federal de Minas Gerais - UFMG (NOORDHOEK et al., 2005). A proposta tem como premissa que a promoção da saúde consiste na oferta de atividades dirigidas à transformação dos comportamentos dos indivíduos, focando nos seus estilos de vida (BUSS, 2000).

Em estudo anterior, Noordhoek et al. (2004), descrevem que a maioria dos participantes encontrase acima dos 60 anos (53,33\%), são do sexo feminino $(93,33 \%)$ e recebem até um salário mínimo $(59,99 \%)$. No que se refere ao estado geral da saúde, todos os indivíduos foram diagnosticados com artrite reumatóide; 46,66\% apresentam um tempo de evolução da doença entre 10 e 20 anos e possuem uma ou mais doenças associadas, estando presente o quadro de hipertensão arterial em 46,66\% dos participantes.

O método de intervenção grupal em terapia 
ocupacional favorece o conhecimento acerca da doença e de seus impactos funcionais, o esclarecimento sobre modalidades de tratamento, a obtenção de orientações e a adoção de comportamentos favoráveis à proteção das articulações e à conservação de energia corporal. Além disso, propicia o convívio e a socialização de indivíduos que possuem doenças semelhantes (MELVIN, 1982). No GOIADR, o processo educacional ocorre ao mesmo tempo em que atividades lúdico-recreativas são desenvolvidas, numa constante estimulação à participação, para com isso, levar o indivíduo à aquisição de um papel mais ativo frente à sua doença. No projeto, o processo terapêutico ocupacional é colaborativo e centrado no cliente (SUMSION, 2003), buscando a promoção da saúde através do engajamento em ocupações propositadas e significativas, escolhidas em conjunto com a equipe e os participantes. Existe a valorização das capacidades remanescentes e o estímulo à descoberta de potencialidades entre os indivíduos, buscando-se associar as expectativas e demandas pessoais. Dessa forma, retira-se o foco da doença, valorizando a singularidade do sujeito. Uma das ferramentas mais frequentemente usadas são atividades lúdico-recreativas, pois acredita-se que as mesmas são recursos eficientes na promoção de alegria, relaxamento, alongamento, autopercepção, reconhecimento e consciência corporal, o que subsidia o enfrentamento das condições de saúde. Os participantes apontam quais atividades e temas a serem desenvolvidos.

Atualmente, uma média de quinze indivíduos participam das atividades. No projeto, os encontros são sempre grupais, com freqüência semanal e duração de uma hora e meia. O grupo é aberto à entrada de novos membros e aos cuidadores, familiares e membros da comunidade do participante.

A equipe do projeto é interdisciplinar, composta por diversos profissionais da área da saúde, como terapeutas ocupacionais, reumatologistas, nutricionistas, psicólogos e musicoterapeutas.

\section{O setting grupal: uma estratégia pedagógica}

O homem é um ser essencialmente social. Ao longo da vida, assumirá vários papéis e pertencerá a distintos grupos. Com o decorrer do processo de envelhecimento, as redes sociais vão se tornando esparsas, na mesma medida em que se reduz as oportunidades de convívio de indivíduos maduros e idosos.

Para Law (2002), a presença de incapacidade pode levar a uma participação menos diversificada na vida, fazendo com que o indivíduo apresente um reduzido engajamento nas redes sociais e um menor envolvimento em atividades recreativas. Nesse aspecto, Doeglas et al. (1994), Pedretti e Early (2005), registram o suporte social como sendo uma estratégia capaz de melhorar a capacidade de enfrentamento da doença e a percepção da qualidade de vida de indivíduos com doenças reumáticas. Sob essa premissa, Newman e Mulligan (2000), realizaram uma revisão bibliográfica dos fatores psicossociais associados às doenças reumáticas e constataram que, vários dentre os estudos comparados, relataram um impacto positivo do suporte social no bem estar dos indivíduos.

Gáspari (2005) aponta para a importância do cenário educativo grupal para indivíduos idosos ao considerar que, no coletivo, existe a oportunidade de o sujeito colocar-se frente a frente com fatos, idéias e realidades, favorecendo a incorporação de novas atitudes.

Para Holman e Lorig (1997), no processo de educação do paciente reumático, o sujeito é o principal ator e também um crucial avaliador. Nesse sentido, as atividades realizadas em grupo têm caráter dinâmico, são baseados numa demanda e escolhidas de acordo com as necessidades, interesses e sugestões apresentadas pelos participantes (NOORDHOEK, 2005).

\section{O emprego de atividade lúdico-recreativa no GOIADR}

Viver uma vida significativa e agradável é essencial para o bem-estar. Segundo Trombly (2005), somente as ocupações significativas permanecem no repertório de uma pessoa ao longo da vida. Taylor (2003) acrescenta que as atividades de lazer contribuem para a aquisição de um estilo de vida saudável, o desenvolvimento da identidade, a socialização, o descanso, a expressão criativa e a promoção da saúde.

As atividades desenvolvidas no projeto são idealizadas em conjunto com os participantes e essencialmente lúdicorecreativas. Acontecem nos moldes de oficinas terapêuticas que, de acordo com Afonso (2000), utilizam informações e reflexões acerca de um ou mais temas e trabalham com os significados afetivos e as vivências relacionadas com o(s) tema(s) proposto(s).

Para Kielhofner (1997), o uso de ocupações propositadas e significativas como estratégia de intervenção agrega valor ao tratamento, podendo repercutir positivamente nos resultados. A abordagem do paciente reumático através de atividades lúdico-recreativas tem por objetivo intervir na experiência da saúde e no processo de adoecimento vivenciado pelos indivíduos.

É descrito na literatura o benefício e a repercussão 
NOORDHOEK, J. et al. Relato de experiência da atuação. Rev. Ter. Ocup. Univ. São Paulo, v. 20, n. 1, p. 13-19, jan./abr. 2009.

dessas atividades na saúde física e mental dos praticantes. De acordo com Zimerman (2000), atividades corporais e artísticas, ao serem realizadas em grupo, proporcionam socialização, diálogo, respeito e apoio mútuos, motivação, cooperação, partilha e senso crítico frente à realidade. Esses fatores, associados, terão como conseqüência o crescimento pessoal dos participantes. Miranda e Godeli (2003) registram que a música proporciona uma experiência sensorial, afetando o estado afetivo-emocional do ouvinte. Para Leão e Silva (2005), a música é capaz de influenciar o componente psicológico e fisiológico da dor, podendo ser usada como uma medida complementar no controle da dor crônica. A dança, atividade que envolve música e exercício físico pode ser usada despertando o lúdico na vivência do cotidiano dos participantes (SANTOS, 2001). É uma atividade de fácil aplicação, possui um baixo risco de acidentes e proporciona um grande benefício físico e social. (KUDLACEK et al., 1997). Dessa forma, a dança atinge todos os domínios do comportamento humano: perceptivo, sensório-motor e cognitivo.

No projeto, a intervenção inicia com os participantes sentados em roda. Nesse momento, o condutor (sempre um terapeuta ocupacional), estimula a proposição de temas a serem discutidos e a sugestão de atividades. Busca-se colher informações sobre o modo como os indivíduos estão vivenciando a doença e desempenhando suas atividades cotidianas, e o conhecimento deles acerca de métodos de proteção das articulações e uso de tecnologias assistivas, como splints e adaptações. Em seguida, atividades lúdicorecreativas são desenvolvidas objetivando ampliar a consciência corporal dos participantes (possibilitando a realização de tarefas de modo adequado e harmonioso), estimular a convivência, a expressão de vivências e a movimentação dos membros (buscando prevenir a rigidez articular e retardar a instalação de deformidades). Ao final do encontro, os indivíduos sentam-se novamente em roda, onde trocam relatos sobre o que acabaram de experenciar.

Exemplos de atividades desenvolvidas são: jogos de mímica, dança sênior, movimentações com música e com uso de bastões e bambolês, jogos com balões e cordas, contos de histórias e brincadeiras de roda. Em todas elas, são incentivadas a movimentação ativa dos membros e a interação entre os participantes. No decorrer do encontro, é observada a postura de cada indivíduo. O condutor do grupo procura adaptar e graduar as atividades à condição física e emocional dos sujeitos, oferecendo o suporte necessário àqueles que apresentam dificuldades em realizá-las. No intervalo de cada atividade, são feitos esclarecimentos sobre os princípios e métodos de proteção articular e conservação de energia, a artrite reumatóide e seus impactos físicos e funcionais, e sobre a necessidade de utilização de splints e adaptações de utensílios para a manutenção da integridade articular, melhora da função e prevenção de deformidades.

No projeto, as atividades lúdico-recreativas são desenvolvidas de modo planejado. A cada encontro, são propostas de 2 a 3 atividades, conforme as demandas levantadas pelo grupo no encontro anterior. Em seguida, os participantes escolhem a seqüência na qual as atividades serão desenvolvidas.

$\mathrm{O}$ trabalho em equipe multidisciplinar acontece em dois momentos: no planejamento das atividades, onde se busca uma interlocução entre os profissionais vinculados ao projeto, e na identificação da necessidade do grupo de um maior aprofundamento em assuntos não específicos da terapia ocupacional, como por exemplo, os tratamentos cirúrgico e medicamentoso e o acompanhamento nutricional.

\section{PROCEDIMENTOS}

Para identificarmos a influência das atividades lúdico-recreativas no grupo de orientação, foi realizada a observação dos indivíduos antes, durante e logo após a intervenção. A observação perdurou por dois anos seguidos. Registrou-se depoimentos dos participantes, proferidos espontaneamente, e fatos relevantes durante o desenvolvimento das atividades, tais como repercussões do grupo na vida dos sujeitos, a influência do aprendizado adquirido no desempenho das atividades cotidianas, o nível de envolvimento no grupo (a interação com os colegas), as expressões corporais e o modo com que cada indivíduo reagia às atividades propostas em termos de movimento corporal e de postura social.

\section{RESULTADO}

Ao observarmos os indivíduos ao longo do tempo, foi possível perceber e ouvir dos mesmos, relatos de sentimentos que acompanham o processo de transformação do participantes, a descoberta do autoconhecimento, da condição de se tornar ativo frente a sua própria condição de saúde. Depoimentos de autopercepção e inserção em um grupo com absoluta aceitação de suas habilidades e limitações. Pouco a pouco os participantes se mostravam menos inibidos, menos sisudos, mais alegres e com maior participação social. Em várias oportunidades ouviu-se dos participantes relatos de uma melhora na percepção da qualidade de vida dos mesmos. Alguns referem nunca terem tido, anteriormente, oportunidades de vivenciar 
atividades lúdicas, recreativas e com tanto aprendizado.

O projeto tem empreendido esforços no sentido da promoção da saúde de indivíduos reumáticos. Os registros realizados durante a vigência do projeto possibilitaram-nos colher importantes informações acerca de como as atividades lúdico-recreativas influenciavam os participantes. Identificamos que, ao final de cada encontro, os indivíduos demonstravam uma maior movimentação dos membros, apresentavam-se dispostos ao contato com os colegas de grupo, falavam abertamente de si e do processo que estavam vivenciando. Além disso, o desempenho nas atividades diárias foi favorecido através do aumento do conhecimento dos indivíduos acerca da doença, da possibilidade de gerenciamento dos sintomas e das estratégias de proteção articular e de conservação de energia.

\section{DISCUSSÃO}

A singularidade da terapia ocupacional é considerar a ocupação como central na promoção e manutenção da saúde e bem-estar dos indivíduos (LAW, 1998). Alcançar a melhora da participação nas atividades e em papéis rotineiros, modificando assim, o status de saúde desses indivíduos, é o seu objetivo nas diferentes áreas de atuação da profissão (TROMBLY, 2005). Para Melvin (1982), o trabalho com indivíduos reumáticos tem como premissa o desenvolvimento das potencialidades individuais através da descoberta e/ou aprimoramento das habilidades pessoais, auto-estima e satisfação no viver. O foco do tratamento deve estar na criação de estratégias que permitam manter ou melhorar a independência funcional, a participação social e a qualidade de vida dos sujeitos (MELVIN, 1982).

Estudos realizados por Barry et al. (1994) e Barlow et al. (1998) são exemplos de programas educacionais baseados no aprendizado individual do paciente reumático. Porém, a grande maioria dos estudos investiga a abordagem grupal, como por exemplo, Hammond et al. (1994), Lindroth et al. (1997) e Helliwell et al. (1999). Esses comprovaram a eficácia do cenário grupal e descreveram a importância de as atividades serem desenvolvidas de forma contextualizada, associando os conteúdos abordados à análise de necessidades e às expectativas individuais, fomentando discussões entre os participantes. No GOIADR, a identificação de fatores ocupacionais que afetam a saúde e o bem-estar dos indivíduos, a avaliação dos problemas sociais envolvidos nas doenças reumáticas e a preocupação em se criar uma educação voltada para a conquista da autonomia e da independência na execução de tarefas, através do estímulo à auto-eficácia dos participantes, vão ao encontro da contextualização proposta pelos referidos autores.

Hammond etal.(1999) considera crucial a participação de familiares em programas educacionais acreditando com isso, na extensão e manutenção dos resultados obtidos. Além dos familiares, o Grupo de Orientação aos Indivíduos Acometidos por Doenças Reumáticas encoraja e estimula a participação de cuidadores e de outros membros da comunidade na qual o indivíduo está inserido. A experiência ora relatada, demonstra que a parceria entre os profissionais, os indivíduos acometidos por doenças reumáticas e a rede social, funciona como uma "caixa de ressonância", expandindo a aquisição do conhecimento e favorecendo a conscientização sobre as doenças reumáticas e seus impactos funcionais, contribuindo para a incorporação de hábitos de vida saudáveis e para a mudança de comportamentos no manejo do indivíduo reumático.

Galheigo (1999) conceitua a equipe interdisciplinar, referindo-se ao seu propósito de promover o diálogo entre os conteúdos e métodos dos profissionais que a integram. A equipe interdisciplinar tem agregado qualidade ao trabalho desenvolvido, contribuindo para uma abordagem integral do paciente reumático e refletindo a importância de ações conjuntas entre os diversos profissionais da saúde.

\section{CONSIDERAÇÕES FINAIS}

Existe uma alta prevalência de doenças crônicas na população Brasileira. No grupo de doenças reumáticas, a causa geralmente é desconhecida e a cura, até o momento, é inviável (MOREIRA; CARVALHO, 1996). As limitações funcionais geram um significativo impacto na vida dos indivíduos, podendo levá-los a uma participação menos diversificada nas redes sociais e a um menor envolvimento em atividades recreativas (TROMBLY, 2005). A elucidação dos mecanismos envolvidos nas enfermidades reumáticas e o conhecimento da fisiologia da dor crônica, não são suficientes para a compreensão integral dos indivíduos. É necessária a avaliação dos aspectos psicossociais envolvidos, das expectativas, do estilo de vida dos indivíduos, para assim, melhor planejar e intervir junto a essa população. No GOIADR, a Terapia Ocupacional, tendo como foco a promoção da saúde, intervém para o rompimento de isolamento social e da inatividade, promovendo a vivência de atividades prazerosas e significativas, favorecendo a melhora da autoestima, da autonomia e da independência do indivíduo na execução das tarefas cotidianas. Além disso, intervém na aquisição de conhecimento sobre si mesmo e no controle 
sobre sua condição de saúde e no processo de adoecimento vivenciado.

A literatura disponível é incipiente na descrição da aplicação de atividades lúdico-recreativas como método de intervenção em educação para a saúde de pacientes reumáticos. Autilização de atividades lúdico-recreativas no processo educacional tem demonstrado ser uma abordagem efetiva na aquisição de um papel ativo do indivíduo frente ao processo saúde-doença. O relato dessa experiência visa estimular a construção de conhecimentos por meio de uma perspectiva interacional, onde o diálogo é o fio condutor. Soluções são compartilhadas entre os interessados, tendo como pano de fundo, o engajamento dos participantes em atividades que proporcionam bem estar, contribuindo para melhora na percepção da qualidade de vida.

NOORDHOEK, J.; SILVA, M. C. de O.; TORQUETTI, A.; CISNEROS, L. de L. A report of an experience of occupational therapy in a group with individuals with rheumatic diseases. Rev. Ter. Ocup. Univ. São Paulo, v. 20, n. 1, p. 13-19, jan./abr. 2009.

\begin{abstract}
The individual with a rheumatic disease presents physiological and anatomical changes which interfere with performance of the daily activities. Occupational therapy working with health promotion for this population aims to improve functional, social and emotional aspects to help achieve better performance of their daily activities and to promote better quality of life. The aim of this paper is to relate a group of orientation for patients with rheumatic diseases. The proportions are based on empiric experience of the group: "Orientation for individuals with rheumatic diseases" The group is a community based project of The Federal University of Minas Gerais. This educational process offered through therapeutic workshops based on ludic-recreative activities. The strategy enables education, socialization and progress of skills to face the disease and the performance of their daily role in life.
\end{abstract}

KEY WORDS: Health promotion. Occupational therapy. Rheumatic diseases.

\title{
REFERÊNCIAS
}

AFONSO, M. L. Oficina em dinâmica de grupo: um método de intervenção psicossocial. Belo Horizonte: Edições do Campo Social, 2000.

BARLOW, J.; WRIGHT, C. C. Knowledge in patients with rheumatoid arthritis: a longer term follow-up of a randomized controlled study of patient education leaflets. Br. J. Rheumatol., v. 37, n. 4, p. 373-376, 1998.

BARLOW, J.; TURNER, A. P.; WRIGTH, C. C. A randomized controlled study of the arthritis self-management programme in the UK. Health. Educ. Res., n. 15, p. 665-680, 2000.

BARRY, M. A.; PURSER, J.; HAZLEMAN, R., et al. Effect of energy conservation and joint protection education in rheumatoid arthritis. Br. J. Rheumatol., n. 33, p. 1171-1174, 1994.

BUSS, P. M. Promoção da saúde e qualidade de vida. Cien. Saúde Coletiva, v.5, n.1, p. 163-177, 2000.

CARVALHO, M. G. R.; NOORDHOEK, J.; SILVA, M. C. O.
Grupo de orientação a indivíduos acometidos por doenças reumáticas: espaço educativo e terapêutico. Rev. Bras. Reumatol., v. 46, n. 2, p. 134-136, 2006.

CARDOSO, T. H. F. Educação do reumático: revisão sobre a atuação da terapia ocupacional e o uso da abordagem em grupo [monografia]. Belo Horizonte: Universidade Federal de Minas Gerais, 2005

CZERESNIA, D. The concept of health and the difference between prevention and promotion. Cad. Saude Publica, v. 15, n. 4, p. 701-709, 1999.

DOEGLAS, D.; SUURMEIJER, T.; KROL, B.; et al. Social suport, social disability, and psychological well-being in rheumatoid arthritis. Arthritis Care Res., v. 7, n. 1, p. 10-15, 1994.

GALHEIGO, S. M. A transdisciplinaridade enquanto princípio e realidade das ações de saúde. Rev. Ter. Ocup. Univ. São Paulo, v. 10, n. 2/3, 1999 .

GÁSPARI, J. C.; SCHWARTZ, G. M. O Idoso e a ressignificação 
emocional do lazer. Psicol.: Teoria Pesq., v. 21, n. 1, p. 69-76, 2005.

HAHN, M. S. Promoção de saúde e terapia ocupacional. Rev. Centro Est. Ter. Ocup., v. 1, n. 1, p. 10-13, 1995.

HAMMOND, A. Joint protection behavior in patients with rheumatoid arthritis following an education program. Arthritis Care Res., v. 7. n. 1, p. 5-9, 1994.

HAMMOND, A.; FREEMAN, K. One year outcomes of a randomized controlled trial of an educational-behavioural joit protection programme for people with rheumatoid arthritis. Rheumatology, n. 40, p. 1044-1051, 2001.

HAMMOND, A.; LONCOLN, N.; SUTCLIFFE, L. A. A crossover trial evaluating an educational-behavioural joint protection programme for people with rheumatoid arthritis. Patient Educ. Cous., n. 37, p. 19-32, 1999.

HELLIWELL, P. S.; O'HARA, M.; HOLDSWORTH, J.; et al. A 12-month randomized controlled trial of patient education on radiographic changes and quality of life in early rheumatoid arthritis. Br. Soc. Rheumatol., 1999.

HOLMAN, H. R.; LORIG, K. Patient education: essencial to good health care for patients with chronic arthritis. Arthritis Rheum., n. 40, v. 8, p. 1371-1373, 1997.

KIELHOFNER, G. Conceptual foundations of occupational therapy. 2. ed. Philadelphia: Davis Company, 1997.

KUDLACEK, S.; PIETSCHMAN, F.; BERNECKER, P. The impact of a senior dancing program on spinal and peripheral bone mass. Am. J. Phys. Med. Rehabil., n. 76, v. 6, nov. 1997.

LAW, M. Participation in the occupations of everyday life. Am. J. Occup. Ther., v. 56, n. 6, p. 640-649, 2002.

LAW, M.; STEINWENDER, S.; LECLAIR, L. Occupation, health and well-being. Can. J. Occup.Ther., v. 65, n. 2, p. 81-91, 1998.

LEÃO, E. R.; SILVA, M. P. J. The relationship between music and musculoskeletal chronic pain. Online Bras. J. Nursing [periódico on line]. v. 4, n.1, 2005. Disponível em: $<$ http:// www. uff.br/nepae/objn401leaoetal.htm[2006jul22>. Acesso em: 10 jun. 2007.

LINDROTH, Y.; BRATTSTROM, M.; BELLMAN, I., et al. A problem-based education program for patients with rheumatoid arthritis: evaluation after three and twelve months. Arthritis Care Res., v. 10, n. 5, p. 325-332, 1997.

MELVIN, J. L. Rheumatic disease: occupational therapy and rehabilitation. 2. ed. Philadelphia: Davis Company, 1982.

MIRANDA, M. L. J.; GODELI, M. R. C. S. Música, atividade física e bem-estar psicológico em idosos. Rev. Bras. Ci. Mov., v.
11. n. 4, p. 87-94, 2003.

MOREIRA, C.; CARVALHO, M. A. P. Noções práticas de reumatologia. Belo Horizonte: Health, 1996.

NEWMAN, S.; MULLIGAN, K. The psychology of rheumatic diseases. Bailliere's Clin. Rheumatol., v. 14, n. 4, p. 773-786, 2000 .

NOORDHOEK, J.; GOULART, F.; LOSCHIAVO, F. Q. Estudo descritivo dos pacientes atendidos no projeto de extensão de orientação aos indivíduos acometidos por doenças reumáticas. In: CONGRESSO BRASILEIRO DE EXTENSÃO UNIVERSITÁRIA, Belo Horizonte, 12-15 set. 2004. Anais... Belo Horizonte, 2004.

NOORDHOEK, J.; SILVA, M. C. O.; CARVALHO, M. G. Terapia Ocupacional: relato de experiência em grupo de orientação a indivíduos acometidos por doenças reumáticas. In: ENCONTRO DE EXTENSÃO DA UFMG, 7., Belo Horizonte, 2005. Anais... Belo Horizonte: Universidade Federal de Minas Gerais, 2005.

PEDRETTI, L. M.; EARLY, M. B. Terapia ocupacional capacidades práticas para as disfunções físicas. São Paulo: Roca, 2005.

POLLEY, H. F.; HUNDER, G. G. Anamnese reumatológica e exame físico das articulações. Rio de Janeiro: Interamericana, 1980.

SANTOS, T. M. A. A dança como força de expressão no lazer [monografia]. Belo Horizonte: Universidade Federal de Minas Gerais, 2001.

SHOLTEN, C.; BRODOWICZ, T.; GRANINGER, W.; et al. Persistent functional and social benefit 5 years after a multidisciplinary arthritis training program. Arch. Phys. Med. Rehabil., n. 80, p. 1282-1287, 1999.

SOUZA, E. M.; GRUNDY, E. Promoção da saúde, epidemiologia social e capital social: inter-relações e perspectivas para a saúde pública. Cad. Saude Publica, v. 20, n. 5, p. 1354-1360, 2004.

SUMSION, T. Prática baseada no cliente na terapia ocupacional: Guia para implementacão. São Paulo: Roca, 2003.

TAAL, E.; RASKER, J. J.; WIEGMAN, O. Group education for rheumatoid arthritis patients. Semin. Arthritis Rheum., v. 26, n. 6, p. 805-816, 1997.

TAYLOR, J. Women`s leisure activities, their social stereotypes and some implications for identity. Br. J. Occup. Ther., v. 66, n. 4, p. 151-158, 2003

TROMBLY, C. Terapia ocupacional para disfunções físicas. Rio de Janeiro: Santos Editora, 2005.

ZIMERMAN, G. I. Velhice: aspectos biopsicossociais. Porto Alegre: Artmed Editora, 2000. 